\title{
Molecular Imaging of Immunotherapy Targets in Cancer
}

\author{
Emily B. Ehlerding*1 ${ }^{*}$ Christopher G. England*1, Douglas G. McNeel ${ }^{2,3}$, and Weibo Cai ${ }^{1,3,4}$ \\ ${ }^{1}$ Department of Medical Physics, University of Wisconsin-Madison, Madison, Wisconsin; ${ }^{2}$ Department of Medicine, University of \\ Wisconsin-Madison, Madison, Wisconsin; ${ }^{3}$ University of Wisconsin Carbone Cancer Center, Madison, Wisconsin; and ${ }^{4}$ Department \\ of Radiology, University of Wisconsin-Madison, Madison, Wisconsin
}

\begin{abstract}
Immunotherapy has emerged as a promising alternative in the arsenal against cancer by harnessing the power of the immune system to specifically target malignant tissues. As the field of immunotherapy continues to expand, researchers will require newer methods for studying the interactions between the immune system, tumor cells, and immunotherapy agents. Recently, several noninvasive imaging strategies have been used to map the biodistribution of immune checkpoint molecules, monitor the efficacy and potential toxicities of the treatments, and identify patients who are likely to benefit from immunotherapies. In this review, we outline the current applications of noninvasive techniques for the preclinical imaging of immunotherapy targets and suggest future pathways for molecular imaging to contribute to this developing field.
\end{abstract}

Key Words: PD-1; PD-L1; CTLA-4; immune checkpoints; immunotherapy; positron emission tomography (PET)

J Nucl Med 2016; 57:1487-1492

DOI: 10.2967/jnumed.116.177493

Although the field of cancer immunotherapy has undergone tremendous growth during the past decade, the idea of using a person's own immune system to combat disease can be traced back over a century $(1,2)$. These approaches have immense implications for oncology applications, yet clinical translation of immunotherapy is currently limited. In the past, concerns over potential immune-related adverse effects, as well as limited benefit from traditional vaccination techniques, limited the clinical use of immunotherapies; however, the recent Food and Drug Administration (FDA) approval of several immune checkpoint inhibitor immunotherapies has led to renewed interest in the field. To date, the 3 primary targets of checkpoint inhibition include the programmed death protein-1 receptor (PD-1), its ligand (programmed death ligand-1 [PD-L1]), and the cytotoxic T-lymphocyte-associated antigen-4 receptor (CTLA-4). As summarized in Table 1, several antibody-based checkpoint inhibitors have received

Received May 19, 2016; revision accepted Jul. 25, 2016.

For correspondence or reprints contact: Weibo Cai, 1111 Highland Ave., Room 7137, Madison, WI 53705.

E-mail: wcai@uwhealth.org

${ }^{*}$ Contributed equally to this work.

Published online Jul. 28, 2016.

COPYRIGHT (c) 2016 by the Society of Nuclear Medicine and Molecular Imaging, Inc.
FDA approval, with many others currently in various stages of clinical trials.

The innate immune system contains several checkpoints that ensure immune cells capable of recognizing self-antigens do not destroy healthy tissues. Thus, as tumors are selfderived tissues, they often display these same antigens and avoid immune surveillance (1). By interrupting these immune checkpoints that have been hijacked by tumors, checkpoint blockade therapy allows the immune system to recognize tumor-associated antigens and consequently destroy these malignant cells (2). Interested readers are referred to the review by Buchbinder et al. for further information on the mechanisms of these pathways (3). Blockade of these immune checkpoint pathways has shown incredible efficacy in the treatment of many cancers, including Hodgkin lymphoma, non-small cell lung cancer, melanoma, and others. In some instances, a correlation has been found between receptor expression and the efficacy of immune interventions (4); however, this expression seems to be dynamic and heterogeneous, and as a result, fixed immunohistochemical analyses may not provide accurate information at the time of treatment (5). Molecular imaging can provide nearly realtime information about receptor expression levels, allowing physicians to predict which patients may benefit from immunotherapy and accounting for response differences between individual patients. In return, these prescreening measures not only will spare patients ineffective therapy and potential adverse effects but also will have economic implications, as cancer therapy remains costly and timeintensive (6). In addition to patient stratification, molecular imaging of immunotherapy targets may provide insight into the status of the immune system and overall disease progression.

Since ${ }^{18}$ F-FDG PET monitors cellular metabolism and immunotherapy elicits a natural inflammatory response, traditional PET imaging using ${ }^{18} \mathrm{~F}$-FDG has proven inadequate in examining responses to immunotherapy (7). Nonetheless, immunotherapy patients are still routinely examined with ${ }^{18}$ F-FDG, causing disease monitoring to be inherently subjective. To date, no clinical trials have been performed for molecular imaging of immune checkpoint targets. However, several preclinical trials have shown that noninvasive imaging techniques using PET, SPECT, and optical imaging are viable platforms for determination of receptor density and hold 
TABLE 1

FDA Approval Status of Immune Checkpoint Inhibitors for Treatment of Cancer

\begin{tabular}{|c|c|c|c|c|}
\hline Target & Agent & Application & Status & Reference \\
\hline \multirow[t]{5}{*}{ PD-1 } & Nivolumab & Melanoma & Approved & (12) \\
\hline & & Lung cancer & Approved & (36) \\
\hline & & Renal cell cancer & Approved & (37) \\
\hline & Pembrolizumab & Melanoma & Approved & (11) \\
\hline & & Lung cancer & In phase I clinical trials & (38) \\
\hline \multirow[t]{2}{*}{ PD-L1 } & Atezolizumab & Lung cancer & In phase II clinical trials & (39) \\
\hline & & Bladder cancer & In phase II clinical trials & $(40)$ \\
\hline \multirow[t]{2}{*}{ CTLA-4 } & Tremelimumab & Melanoma & In phase III clinical trials & (26) \\
\hline & Ipilimumab & Melanoma & Approved & (25) \\
\hline
\end{tabular}

promise for stratification of patients for future immune therapies. In addition, by tracking the fate of immune cells in vivo, researchers may be able to monitor adverse effects related to the off-target accumulation of antibodies and elucidate the mechanisms that underlie these restrictive toxicities. Molecular imaging techniques thus have nearly limitless potential to revolutionize the emerging field of immunotherapy.

\section{PD-1}

PD-1 is expressed on the surface of T cells, B cells, and natural killer cells and acts as a negative regulator of T-cell activity (8). PD-1 expression was also recently reported in certain subpopulations of melanoma cells (9). PD-1 may interact with one of its two endogenous ligands, PD-L1 and PD-L2, which are found on the surface of antigenpresenting cells or tumor cells. On PD-1 interaction, a kinase signaling pathway that normally results in T-cell activation is inhibited; thus, immunotherapy strategies that interfere with the PD-1 checkpoint have shown enhanced anticancer activity in the clinic. There are currently two FDA-approved antibodies targeting PD-1: pembrolizumab (Keytruda; Merck \& Co., Inc.) and nivolumab (Opdivo; Bristol-Myers Squibb) (10). Although these two antibodies have the same biologic target, their binding affinities and production source are different. The binding affinity of pembrolizumab $(<100 \mathrm{pM})$ for $\mathrm{PD}-1$ is nearly 10 -fold higher than that of nivolumab $(3 \mathrm{nM})$. The source of production also differs, as pembrolizumab is a humanized murine antibody whereas nivolumab is a fully human antibody. Despite these differences, the objective response rates in advanced melanoma were similar between pembrolizumab (26\%-38\%) and nivolumab (31\%-40\%) (10), suggesting that the therapeutic dosages may be saturating the receptor in therapy. PD-1-targeted treatments have shown fewer adverse effects than treatments using the CTLA-4 pathway; however, significant immune-mediated responses were still reported during clinical trials $(11,12)$.

Imaging techniques targeting PD-1 are unique because, in most cases, they do not allow visualization of the primary tumor; rather, PD-1-targeted probes allow for imaging of PD-1 expressed on immune cells. For example, Natarajan et al. developed a PET tracer to image PD-1-expressing tumor- infiltrating lymphocytes (TILs) (13). The murine anti-PD-1 monoclonal antibody was prepared with DOTA for radiolabeling with ${ }^{64} \mathrm{Cu}$ (half-life [ $\left.\mathrm{t}_{1 / 2}\right], 12.7 \mathrm{~h}$ ). The biodistribution of the tracer was mapped in a transgenic mouse model of melanoma. The tracer effectively accumulated in the tumor with a percentage injected dose per gram (\% ID/g) of $7.4 \pm 0.71$ at $48 \mathrm{~h}$ after injection, resulting in a tumor-tomuscle ratio of approximately 11 (Fig. 1A). Blocking studies using excess antibody decreased tumor uptake to $4.51 \pm$ $0.26 \% \mathrm{ID} / \mathrm{g}$ at $48 \mathrm{~h}$ after injection (Fig. 1A), which further validated the tracer's high specificity for PD-1. Additionally, bioluminescent imaging of luciferase-transfected TILs confirmed that the tumor uptake was due to lymphocyte infiltration of the tumor (Fig. 1B), showing that PET may be used for the noninvasive imaging and determination of PD-1 expression in vivo.

In another study, Hettich et al. developed novel radiotracers for PET imaging of both PD-1 and PD-L1 (14). Melanoma tumor-bearing mice were locally irradiated to induce TIL infiltration into the tumor, most of which are CD8-positive effector cytotoxic lymphocytes uniformly expressing PD-1. The researchers used a ${ }^{64} \mathrm{Cu}$-labeled anti-PD-1 tracer for PET imaging and found that the tracer had increased uptake in the tumor, consistent with higher CD8-positive TIL levels. Uptake could also be reduced by blocking the antigen (Fig. 1C). Furthermore, the authors used a PD-L1 imaging tracer, which will be discussed in the PD-L1 section.

The clinically approved antibody pembrolizumab was also recently evaluated in several murine models, including mice engrafted with human peripheral blood mononuclear cells (15). The ${ }^{89} \mathrm{Zr}$-labeled tracer was found to colocalize with human T-cells, allowing direct visualization of their distribution in vivo.

\section{PD-L1}

In contrast to PD-1, PD-L1 is naturally expressed on several tissues, including some tumor cells, vascular endothelium, hepatocytes, mesenchymal stem cells, T and B cells, macrophages, and mast cells (16). Immune-privileged tissues such as the eye and placenta also display high levels of PD-L1. Blockade of PD-L1 leads to enhanced T-cell activity and inhibition of immune-mediated tumor evasion $(16,17)$. Because 


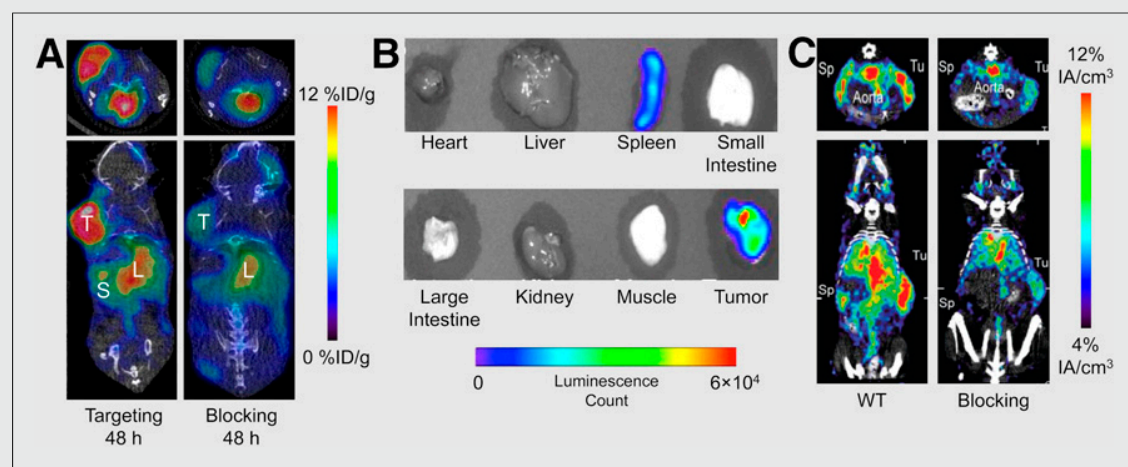

FIGURE 1. Molecular imaging of PD-1-expressing TILs. (A) Subcutaneous melanoma xenografts could be visualized on injection of ${ }^{64} \mathrm{Cu}$-labeled anti-PD-1 antibody. Specificity of tracer was confirmed through blocking study. (Adapted with permission of (13).) (B) Bioluminescence imaging of luciferase-transfected PD-1-expressing $T_{\text {reg }}$ cells validated binding and tumor infiltration of $\mathrm{T}$ lymphocytes. Adapted with permission of (13). (C) PET imaging of melanoma tumor-bearing mice was performed $24 \mathrm{~h}$ after injection with ${ }^{64} \mathrm{Cu}-\mathrm{NOTA}-\mathrm{PD}-1$ antibody. For blocking study, mice were injected with cold antibody $24 \mathrm{~h}$ before tracer injection. IA = injected activity; $\mathrm{Sp}=$ spleen; $\mathrm{Tu}=$ tumor; $\mathrm{WT}=$ wild-type. (Adapted with permission of (14).)

PD-L1 is often expressed on the actual tumor cells, models for imaging this target have proven to be more attainable than those for targets that are expressed only on immune cells. PD-L1 expression is often heterogeneous even within a given cancer subtype, and immunohistochemistry techniques are susceptible to errors as a result (5). Of note, treatment with pembrolizumab requires more than $1 \%$ of non-small cell lung cancer cells to be PD-L1 positive, so a PD-L1 tracer may also find application in the planning of these treatments. Consequently, several anti-PD-L1 imaging agents have been developed to quantify PD-L1 expression in preclinical settings.

Maute et al. engineered a high-affinity PD-1 nonantibody variant for both immuno-PET imaging and immunotherapy in a colon cancer model (18). Rather than using an agent that behaves like PD-L1 and binds to PD-1, the group used directed evolution by yeast-surface display to create a highaffinity (110 pM) competitive antagonist of human PD-L1. Genetically engineered CT26 colon cancer models that were either PD-L1-negative or PD-L1-positive were used for tumor treatment studies, where the PD-L1 antagonist was shown to decrease tumor growth out to 2 wk. Additionally, conjugation of the antagonist with ${ }^{64} \mathrm{Cu}$-DOTA allowed for PET visualization of distribution in both xenografts, with a nearly 2 -fold increase in signal for the positive tumors over negative as seen in Figure 2A. Thus, this agent shows significant promise in theranostics for PD-L1expressing cancers.

Using the mouse and human cross-reactive antibody MPDL3280A, Chatterjee et al. developed a multimodality SPECT and near-infrared imaging agent for visualizing PDL1-expressing tumors (19). Previously, MPDL3280A was proven effective in clinical trials for non-small cell lung cancer, bladder cancer, melanoma, renal cell carcinoma, and others (20), prompting researchers to use this antibody for imaging of PD-L1 expression. Specifically, Chinese hamster ovary tumor xenografts with and without constitutive stable PD-L1 expression were studied using the ${ }^{111}$ In-labeled antibody $\left(\mathrm{t}_{1 / 2}, 2.8 \mathrm{~d}\right)$, as well as the dye-labeled version. Both studies indicated that relative PD-L1 expression could be determined (Fig. 2B). Also, predosing with cold antibody was found to improve tumor uptake through saturation of receptors on splenocytes and increased blood circulation time. Triplenegative breast cancer xenografts, where PD-L1 expression was not genetically engineered, were also evaluated in this study, with higher reported values of PD-L1 expression corresponding to higher \%ID/g values. These studies indicate that clinically used antibodies may be modified with imaging agents to determine their biodistribution before the administration of therapeutic levels, and patients could ultimately be selected for particular therapies on the basis of these results.

Similarly, Heskamp et al. radiolabeled an anti-PD-L1 antibody with ${ }^{111}$ In for SPECT imaging. The biodistribution of the tracer was mapped in mice bearing subcutaneously implanted breast cancer cells with varying PD-L1 expression levels (21). MDA-MB-231 was found to express PD-L1 in over $90 \%$ of cells, whereas MCF-7 exhibited only minimal expression $(0.1 \%)$. Tracer uptake values were found to correlate with PD-L1 expression levels; thus, this tracer was able to distinguish PD-L1 expression levels within cell lines of the same cancer type.

As previously discussed, Hettich et al. designed two new PET tracers for targeting PD-1 and PD-L1 (14). Mice were subcutaneously implanted with PD-L1-positive and PDL1-knockout cells on opposing flanks. Both in vivo imaging and ex vivo analysis confirmed that uptake of the antiPD-L1 tracer was specific for PD-L1-expressing melanoma tissue, whereas the PD-L1-knockout tumors showed minimal uptake of the tracer (Fig. 2C). Interestingly, the authors noted increased uptake of the PD-L1 tracer in brown adipose tissue, suggesting that this tissue may be immunologically relevant.

In another study, Josefsson et al. developed an ${ }^{111} \mathrm{In}$ labeled murine anti-PD-L1 antibody for SPECT biodistribution studies and potential radioimmunotherapy application (22). Imaging in mice bearing NT2.5 murine mammary xenografts revealed high uptake in the tumor, as well as in the spleen, liver, and thymus. However, spleen uptake was greatly reduced on predosing with cold antibody, from nearly $60 \% \mathrm{ID} / \mathrm{g}$ with pure tracer to $11 \% \mathrm{ID} / \mathrm{g}$ with administration of a 100 -fold excess of antibody. Although this predosing strategy allowed for researchers to clearly visualize the tumors, it also resulted in the administration of 


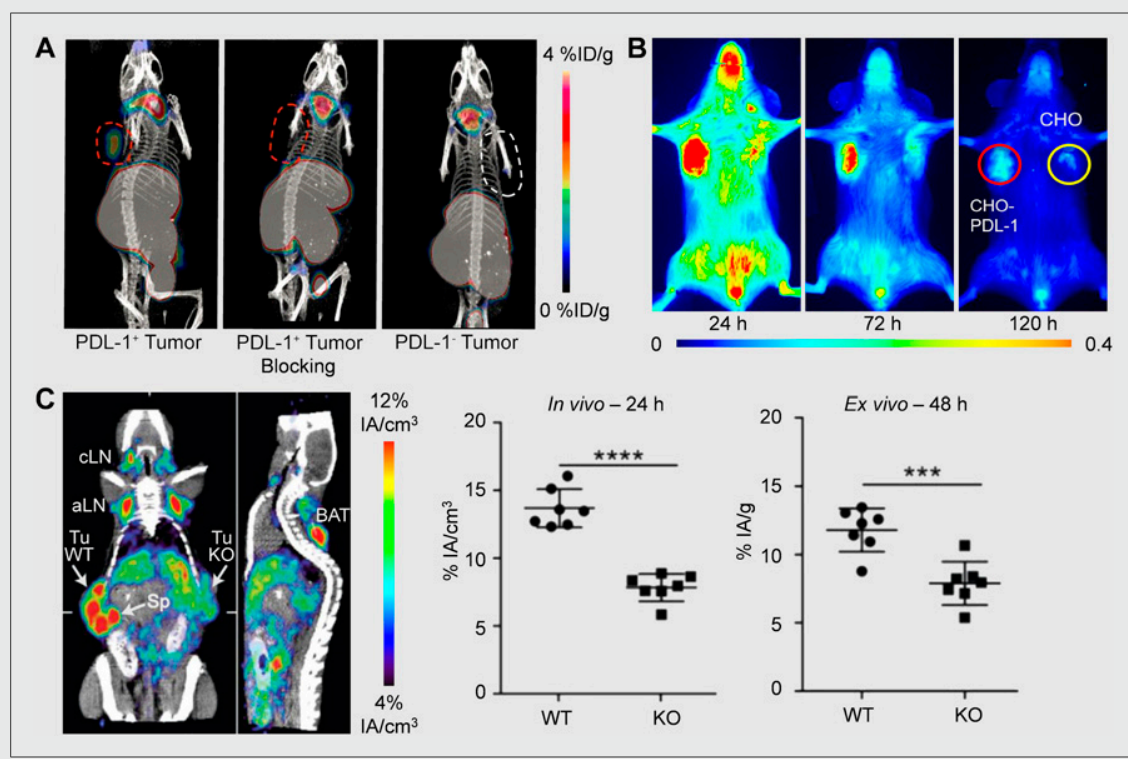

FIGURE 2. Noninvasive imaging of PD-L1 in vivo. (A) ${ }^{64} \mathrm{Cu}$-labeled PD-L1 antagonist allows for distinction between tumors with high and low expression levels of PD-L1 at $1 \mathrm{~h}$ after injection. (Adapted with permission of (18).) (B) Near-infrared images acquired in 800-nm channel show significant differences in uptake of PD-L1 antibody between PDL1-positive and -negative tumors. (Adapted with permission of (19).) (C) PET imaging of mice subcutaneously implanted with PD-L1-positive (WT) or PD-L1-knockout (KO) tumor cells was performed $24 \mathrm{~h}$ after mice were injected with ${ }^{64} \mathrm{Cu}-\mathrm{NOTA}-\mathrm{PD}-\mathrm{L} 1$ antibody. aLN = axillary lymph node; $\mathrm{BAT}=$ brown adipose tissue; $\mathrm{CHO}=$ Chinese hamster ovary; $c L N=$ cervical lymph node; $\mathrm{IA}=$ injected activity; $\mathrm{KO}=$ knockout; $\mathrm{Sp}=$ spleen; Tu = tumor; WT = wild-type. (Adapted with permission of (14).)

high doses of antibody before imaging, a nonideal situation clinically. However, the authors noted that radioimmunotherapy may be performed in conjunction with the traditional antibody-based therapy, and it is in this situation that predosing becomes valuable. Theoretic dosimetry calculations using uptake data from pure tracer injections indicate therapeutic absorbed doses to the tumor using both ${ }^{90} \mathrm{Y}\left(\mathrm{t}_{1 / 2}\right.$, $64.1 \mathrm{~h})$ and ${ }^{177} \mathrm{Lu}\left(\mathrm{t}_{1 / 2}, 6.65 \mathrm{~d}\right)$, but also high spleen doses that may be toxic. Further evaluation of the proposed radioimmunotherapy with this anti-PD-L1 agent is therefore warranted.

\section{CTLA-4}

CTLA-4 is a transmembrane inhibitory receptor expressed on activated $\mathrm{T}$ lymphocytes, including activated $\mathrm{T}$ cells, memory $\mathrm{T}$ cells, and $\mathrm{T}_{\text {reg }}$ cells (23). In addition, it was recently discovered that CTLA-4 may be expressed by many tumor types (24). The interaction between CD80/ CD86 and CTLA-4 expressed on antigen-presenting cells offsets CD28-mediated costimulatory signals, which effectively downregulates T-cell activation through several mechanisms, including raising of the T-cell activation threshold and attenuation of clonal expansion (23). CTLA-4 blockade with antibodies effectively limits this interaction, resulting in enhanced T-cell proliferation and the generation of effector T cells; thus, CTLA-4-targeted antibodies have shown efficacy in the treatment of many cancers. Although targeting of CTLA4 provides therapeutic benefits, antiCTLA-4 therapies can lead to severe adverse effects. Clinical trials have been performed with two anti-CTLA4 agents, ipilimumab (Yervoy; BristolMyers Squibb) (25) and tremelimumab (AstraZeneca) (26), in which tumor response rates for the drugs as monotherapy were approximately $10 \%$. These therapies have been shown to be extremely effective in this subpopulation of patients; thus, molecular imaging may assist in prescreening patients to identify individuals more likely to respond to anti-CTLA-4 immunotherapy.

Recently, PET imaging was used to determine levels of CTLA-4-positive TILs in CT26 colon cancer-bearing mice (27). Here, Higashikawa et al. developed a ${ }^{64} \mathrm{Cu}$-labeled antimouse CTLA-4 antibody to study subcutaneously implanted xenografts in mice (27). The antibody was constructed to recognize the extracellular domain of CTLA-4, similar to the behavior of the FDA-approved antibody ipilimumab. Through this study, the authors verified that CTLA-4 expression on TILs, not the tumor cells themselves, was the cause of tracer uptake, as minimal levels of CTLA-4 were detected in cell cultures or tumors implanted into immunodeficient mice (Fig. 3A). Only when mice were immunocompetent were high CTLA-4 expression levels observed in Western blot analysis of ex vivo tumors. PET imaging evaluation of the antibody indicated slightly higher uptake of the anti-CTLA-4 antibody with respect to a control IgG in immunocompetent models ( $7.5 \% \mathrm{ID} / \mathrm{g}$ vs. $5.8 \% \mathrm{ID} / \mathrm{g}$ at $48 \mathrm{~h}$ after injection), as seen in Figure 3B. Although the anti-CTLA-4 antibody provided enhanced uptake in comparison to the nonspecific antibody, the agent has yet to be evaluated in models where varying levels of CTLA-4 expression are present. However, this first study successfully demonstrated the use of a ${ }^{64} \mathrm{Cu}-$ labeled anti-CTLA-4 antibody to noninvasively image CTLA4 levels on TILs in cancer models.

\section{CHALLENGES AND FUTURE DIRECTIONS}

Visualizing the complex interactions between the immune system and tumor cells can provide vital insight into biomarkers that may be excellent candidates for future immunotherapies. Immunotherapy blockade strategies are becoming more common in the management of several types of cancer; thus, the field is expected to grow exponentially during the next decade. Because immune checkpoint inhibitors rely on adequate receptor expression levels, noninvasive imaging to detect PD-1/PD-L1 or CTLA-4 may allow for enhanced 


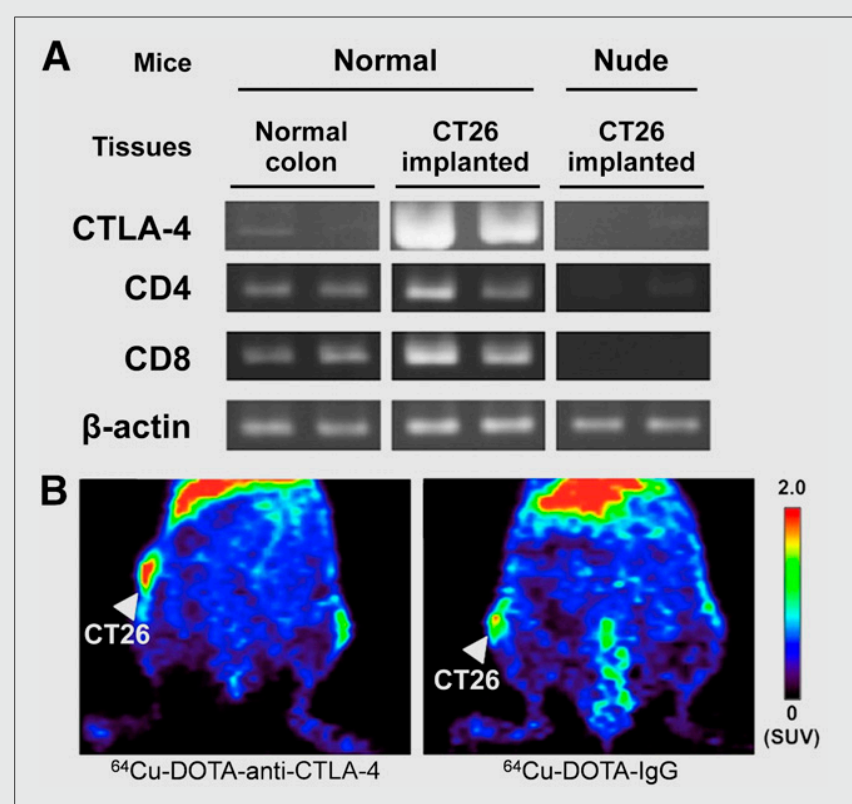

FIGURE 3. PET imaging of colon cancer with ${ }^{64} \mathrm{Cu}$-labeled anti-CTLA-4 antibody. (A) CTLA-4 expression was low in normal colon tissue and absent in CT26 tumors implanted in immunodeficient mice. However, CTLA-4 expression was high in tumors implanted in immunocompetent mice. (B) At $48 \mathrm{~h}$ after injection, ${ }^{64} \mathrm{Cu}$-labeled antibody showed higher uptake in CT26 tumors (left) than did nonspecific antibody control (right). (Adapted with permission of (27).)

patient identification, stratification, and the early assessment of therapeutic response. As many novel immune checkpoints for therapeutic targeting are investigated (1), imaging strategies for prescreening patients will be crucial to the success of these treatments. Additional methods not based on antibody platforms are also being explored, including the in vivo tracking of radiolabeled $\mathrm{T}$ cells (28).

Immunotherapy treatments may also benefit from molecular imaging of targets not directly involved in the checkpoint blockade itself. For instance, CD3 imaging agents may be able to probe the T-cell infiltration occurring during immunotherapy, such as the anti-CTLA-4 therapy studied by Larimer et al. (29). Similarly, Tavare et al. imaged CD8-positive TILs during immune responses to therapy $(30,31)$. These investigations may provide a more universal approach to the monitoring of immunotherapies, as they are not restricted to single immune checkpoint targets.

Because the studies to date in this area have been preclinical, a few steps will be critical for clinical translation. Most of the agents outlined herein are murine antibodies and may not display specificity for human receptors; thus, human or humanized antibodies will need to be created for clinical translation. In addition, humanized or transgenic models used in preclinical research fall short of mimicking the conditions found in human patients. Imaging of these immune checkpoint pathways may also be limited by external conditions, including therapeutic intervention and certain bacterial or viral infections that may modify the immune system (32). As such, it has yet to be seen whether these imaging biomarkers will quantitatively represent the receptor densities.

Many preclinical studies use a predosing strategy with nonlabeled antibody to increase the tracer uptake in targeted tissues of interest (33). Although this certainly provides greater visual contrast between background and malignant tissues, this need to saturate receptor-binding points to one of the critical pitfalls of immunotherapy. Although most biomarkers targeted for imaging are found solely on tumor cells, these immune checkpoint pathway receptors are heterogeneously expressed throughout the entire body, complicating potential imaging strategies. Thus, molecular imaging of an immunotherapy target requires creative approaches that may extend beyond traditional tracer development, as does the generation of adequate preclinical animal models.

Several researchers have proposed that immunotherapy and external radiotherapy may act in synergy to increase the therapeutic indices of these treatments $(34,35)$. Thus, agents that perform well in imaging settings may also be modified in the future with therapeutic nuclides, such as ${ }^{90} \mathrm{Y}$ or ${ }^{177} \mathrm{Lu}$, for combined immunotherapy and targeted radiotherapy, further extending the reach of nuclear medicine into this emerging field.

\section{CONCLUSION}

Cancer immunotherapy blockade interventions have the potential to revolutionize cancer management strategies and are already doing so today. The causes of considerable immune-related adverse effects and limited success rates may be elucidated through the use of noninvasive, neoadjuvant imaging. Molecular imaging of immunotherapy targets such as PD-1, PD-L1, and CTLA-4 has shown promise in preclinical studies, and successful application to the clinic will provide great strides in the fight against cancer. In the future, molecular imaging of immunotherapy targets may enhance patient stratification and provide insight for development of novel immunotherapy targets.

\section{DISCLOSURE}

This work is supported, in part, by the University of Wisconsin-Madison, the National Institutes of Health (NIBIB/NCI 1R01CA169365, R01EB021336, P30CA014520, T32CA009206, and T32GM008505), and the American Cancer Society (125246-RSG-13-099-01-CCE). No other potential conflict of interest relevant to this article was reported.

\section{REFERENCES}

1. Pardoll DM. The blockade of immune checkpoints in cancer immunotherapy. Nat Rev Cancer. 2012;12:252-264.

2. Topalian SL, Drake Charles G, Pardoll Drew M. Immune checkpoint blockade: a common denominator approach to cancer therapy. Cancer Cell. 2015;27:450-461.

3. Buchbinder EI, Desai A. CTLA-4 and PD-1 pathways: similarities, differences, and implications of their inhibition. Am J Clin Oncol. 2016;39:98-106.

4. Halama N, Michel S, Kloor M, et al. Localization and density of immune cells in the invasive margin of human colorectal cancer liver metastases are prognostic for response to chemotherapy. Cancer Res. 2011;71:5670-5677. 
5. Madore J, Vilain RE, Menzies AM, et al. PD-L1 expression in melanoma shows marked heterogeneity within and between patients: implications for anti-PD-1/ PD-L1 clinical trials. Pigment Cell Melanoma Res. 2015;28:245-253.

6. Geynisman DM, Chien C-R, Smieliauskas F, Shen C, Shih Y-CT. Economic evaluation of therapeutic cancer vaccines and immunotherapy: a systematic review. Hum Vaccin Immunother. 2014;10:3415-3424.

7. Gilles R, de Geus-Oei L-F, Mulders PFA, Oyen WJG. Immunotherapy response evaluation with ${ }^{18} \mathrm{~F}$-FDG-PET in patients with advanced stage renal cell carcinoma. World J Urol. 2013;31:841-846.

8. Dolan DE, Gupta S. PD-1 pathway inhibitors: changing the landscape of cancer immunotherapy. Cancer Control. 2014;21:231-237.

9. Kleffel S, Posch C, Barthel Steven R, et al. Melanoma cell-intrinsic PD-1 receptor functions promote tumor growth. Cell. 2015;162:1242-1256.

10. Ivashko IN, Kolesar JM. Pembrolizumab and nivolumab: PD-1 inhibitors for advanced melanoma. Am J Health Syst Pharm. 2016;73:193-201.

11. Tan M, Quintal L. Pembrolizumab: a novel antiprogrammed death 1 (PD-1) monoclonal antibody for treatment of metastatic melanoma. J Clin Pharm Ther. 2015;40:504-507.

12. Topalian SL, Sznol M, McDermott DF, et al. Survival, durable tumor remission, and long-term safety in patients with advanced melanoma receiving nivolumab. $J$ Clin Oncol. 2014;32:1020-1030.

13. Natarajan A, Mayer AT, Xu L, Reeves RE, Gano J, Gambhir SS. Novel radiotracer for immunoPET imaging of PD-1 checkpoint expression on tumor infiltrating lymphocytes. Bioconjug Chem. 2015;26:2062-2069.

14. Hettich M, Braun F, Bartholoma MD, Schirmbeck R, Niedermann G. Highresolution PET imaging with therapeutic antibody-based PD-1/PD-L1 checkpoint tracers. Theranostics. 2016;6:1629-1640.

15. England CG, Ehlerding EB, Hernandez R, et al. Preclinical pharmacokinetics and biodistribution studies of ${ }^{89} \mathrm{Zr}$-labeled pembrolizumab. $J$ Nucl Med. In press.

16. Patel SP, Kurzrock R. PD-L1 expression as a predictive biomarker in cancer immunotherapy. Mol Cancer Ther. 2015;14:847-856.

17. Ahmad SM, Borch TH, Hansen M, Andersen MH. PD-L1-specific T cells. Cancer Immunol Immunother. 2016;65:797-804

18. Maute RL, Gordon SR, Mayer AT, et al. Engineering high-affinity PD-1 variants for optimized immunotherapy and immuno-PET imaging. Proc Natl Acad Sci USA. 2015;112:E6506-E6514.

19. Chatterjee S, Lesniak WG, Gabrielson M, et al. A humanized antibody for imaging immune checkpoint ligand PD-L1 expression in tumors. Oncotarget. 2016;7:10215-10227.

20. Herbst RS, Soria J-C, Kowanetz M, et al. Predictive correlates of response to the anti-PD-L1 antibody MPDL3280A in cancer patients. Nature. 2014;515:563-567.

21. Heskamp S, Hobo W, Molkenboer-Kuenen JDM, et al. Noninvasive imaging of tumor PD-L1 expression using radiolabeled anti-PD-L1 antibodies. Cancer Res. 2015;75:2928-2936.

22. Josefsson A, Nedrow JR, Park S, et al. Imaging, biodistribution, and dosimetry of radionuclide-labeled PD-L1 antibody in an immunocompetent mouse model of breast cancer. Cancer Res. 2016;76:472-479.

23. Buchbinder E, Hodi FS. Cytotoxic T lymphocyte antigen-4 and immune checkpoint blockade. J Clin Invest. 2015;125:3377-3383.
24. Antczak A, Pastuszak-Lewandoska D, Górski P, et al. CTLA-4 expression and polymorphisms in lung tissue of patients with diagnosed non-small-cell lung cancer. BioMed Res Int. 2013;2013:576486.

25. Kim DW, Trinh VA, Hwu W-J. Ipilimumab in the treatment of advanced melanoma: a clinical update. Expert Opin Biol Ther. 2014;14:1709-1718.

26. Ribas A, Kefford R, Marshall MA, et al. Phase III randomized clinical trial comparing tremelimumab with standard-of-care chemotherapy in patients with advanced melanoma. J Clin Oncol. 2013;31:616-622.

27. Higashikawa $\mathrm{K}$, Yagi $\mathrm{K}$, Watanabe $\mathrm{K}$, et al. ${ }^{64} \mathrm{Cu}-\mathrm{DOTA}-A n t i-C T L A-4 \mathrm{mAb}$ enabled PET visualization of CTLA-4 on the T-cell infiltrating tumor tissues. PLoS One. 2014;9:e109866.

28. McCracken MN, Vatakis DN, Dixit D, McLaughlin J, Zack JA, Witte ON. Noninvasive detection of tumor-infiltrating T cells by PET reporter imaging. $J$ Clin Invest. 2015;125:1815-1826.

29. Larimer BM, Wehrenberg-Klee E, Caraballo A, Mahmood U. Quantitative CD3 PET imaging predicts tumor growth response to anti-CTLA-4 therapy. $J$ Nucl Med. May 26, 2016 [Epub ahead of print].

30. Tavare R, Escuin-Ordinas H, McCracken M, et al. Detection of antibody therapyinduced anti-tumor immune responses using anti-CD8 immuno-pet [abstract]. $J$ Immunother Cancer. 2015;3(suppl 2):P391.

31. Tavaré R, Escuin-Ordinas $\mathrm{H}$, Mok S, et al. An effective immuno-PET imaging method to monitor CD8-dependent responses to immunotherapy. Cancer Res. 2016;76:73-82.

32. Das R, Verma R, Sznol M, et al. Combination therapy with anti-CTLA-4 and anti-PD-1 leads to distinct immunologic changes in vivo. J Immunol. 2015;194: 950-959.

33. Boswell CA, Mundo EE, Zhang C, et al. Differential effects of predosing on tumor and tissue uptake of an ${ }^{111}$ In-labeled anti-TENB2 antibody-drug conjugate. J Nucl Med. 2012;53:1454-1461.

34. Sharabi AB, Lim M, DeWeese TL, Drake CG. Radiation and checkpoint blockade immunotherapy: radiosensitisation and potential mechanisms of synergy. Lancet Oncol. 2015;16:e498-e509.

35. Teng F, Kong L, Meng X, Yang J, Yu J. Radiotherapy combined with immune checkpoint blockade immunotherapy: achievements and challenges. Cancer Lett. 2015;365:23-29.

36. Kazandjian D, Suzman DL, Blumenthal G, et al. FDA approval summary: nivolumab for the treatment of metastatic non-small cell lung cancer with progression on or after platinum-based chemotherapy. Oncologist. 2016; 21:634-642.

37. Motzer RJ, Escudier B, McDermott DF, et al. Nivolumab versus everolimus in advanced renal-cell carcinoma. N Engl J Med. 2015;373:1803-1813.

38. Garon EB, Rizvi NA, Hui R, et al. Pembrolizumab for the treatment of nonsmall-cell lung cancer. N Engl J Med. 2015;372:2018-2028.

39. Fehrenbacher L, Spira A, Ballinger M, et al. Atezolizumab versus docetaxel for patients with previously treated non-small-cell lung cancer (POPLAR): a multicentre, open-label, phase 2 randomised controlled trial. Lancet. 2016; 387:1837-1846.

40. Sidaway P. Bladder cancer: Atezolizumab effective against advanced-stage disease. Nat Rev Urol. 2016;13:238. 